\title{
Cloud Asset Pricing Tree (CAPT) Elastic Economic Model for Cloud Service Providers
}

\author{
Soheil Qanbari ${ }^{1}$, Fei $\mathrm{Li}^{1}{ }^{1}$, Schahram Dustdar ${ }^{1}$ and Tian-Shyr Dai ${ }^{2}$ \\ ${ }^{1}$ Distributed Systems Group,Technical University of Vienna, Vienna, Austria \\ ${ }^{2}$ Institute of Finance, National Chiao-Tung University, Hsinchu, Taiwan \\ \{qanbari,li,dustdar\}@dsg.tuwien.ac.at,d88006@csie.ntu.edu.tw
}

Keywords: Cloud Price Elasticity, Asset Pricing, Financial Options, Cloud Federation, Cloud Computing.

\begin{abstract}
Cloud providers are incorporating novel techniques to cope with prospective aspects of trading like resource allocation over future demands and its pricing elasticity that was not foreseen before. To leverage the pricing elasticity of upcoming demand and supply, we employ financial option theory (future contracts) as a mechanism to alleviate the risk in resource allocation over future demands. This study introduces a novel Cloud Asset Pricing Tree (CAPT) model that finds the optimal premium price of the Cloud federation options efficiently. Providers will benefit by this model to make decisions when to buy options in advance and when to exercise them to achieve more economies of scale. The CAPT model adapts its structure to address the price elasticity concerns and makes the demand, price inelastic and the supply, price elastic. Our empirical evidences suggest that using the CAPT model, exploits the Cloud market potential as an opportunity for more resource utilization and future capacity planning.
\end{abstract}

\section{INTRODUCTION}

Cloud providers offer APIs associated with their pool of configurable computing resources (e.g., virtual machines) so that clients can access and utilize them by deploying their packages in runtime environments (Kurze et al., 2011). In a Cloud market, the right to benefit from these pools of Cloud resources with their utilization interfaces, can be delivered as " $\mathrm{On}$ Demand" or "Reserved" instances. For clients, the reserved instances (RIs) are more reliable and economic assets. As a proof, the unit of a resource being studied here is an Amazon EC2 Standard Small Instance (US East) at a price ${ }^{1}$ of $\$ 0.060 /$ hour for an on-demand instance and for a reserved instance, costs $\$ 0.034 /$ hour with an upfront payment of $\$ 61 /$ year, which is almost half price. Therefore, financially, clients are more attracted to RIs. Faced with such dilemma, RIs pose the concern of less future utilization as far as it is not used by either the current or other on-demand clients. This motivates providers to take the opportunity to achieve more resource utilization by keeping all instances in use. Providers may reallocate unused RIs of current owner to other ondemand clients to keep all resources utilized. This

\footnotetext{
${ }^{1}$ http://aws.amazon.com/ec2/pricing/
}

approach makes the RIs unavailable for the current owner. Obviously, it is an obligation for providers to assure the availability of RIs associated with owners, otherwise, lack of resources leads to unmet demands and, while reflecting the SLA violations, leads to financial consequences and penalties. To assure asset availability when lacking resources, providers can seek for more affordable and cost-efficient Cloud open markets to outsource their clients demands. In addition to the fact that Cloud open marketplaces (e.g., Zimory, SpotCloud) and federation offerings (e.g., CloudKick, ScaleUp) offer more resource utilization mechanisms, they also enable further cost reduction due to the market competitive advantage among providers.

The decision to outsource the request to the federation parties is relatively dependent to the asset's price. In a similar model, the Amazon Web Services (AWS) also offer a spot instance pricing model, where the price fluctuates as the market supply and demand changes, and the spot instances will be provisioned to the bidders who won the competition. As soon as the asset's spot price goes above the winning bid, resources will be released. In open Cloud markets, the providers hardly can rely on such mechanism since there is no guarantee as they might lose the resources when the asset price crosses their bid. In order to en- 
courage providers to benefit from the Cloud market, we need a dynamic economic model that keeps resource and financial elasticity sustainably balanced by controlling the asset price oscillation while demand and supply fluctuate. To this end, our contribution is twofold: (i) Analyzing the financial options and pricing elasticity concepts in Cloud federation market. (ii) The flexible pricing model that calculates the optimal premium price of the federation options efficiently and accurately.

The paper continues with a motivation scenario in support of an elastic economic model for pricing Cloud federation assets at section 2. Section 3 presents the basic concepts and preliminaries where the conceptual basis and mathematical models are detailed. Based on this, CAPT pricing model is derived in section 4. We simulate and evaluate our CAPT model and numerical results will be given in section 5 to support the efficiency of our model. Subsequently, section 6 surveys related works. Finally, Section 7 concludes the paper and presents an outlook on future research directions.

\section{MOTIVATION}

Along with elastic resource provisioning, providers may face the limitations and insufficiency of their own resource pool supply. In effect, they can transfer the risk of lacking resources to the federation markets. Federation markets can be of interest for providers as well as for consumers. Clients may profit from lower costs and better performance, while providers may offer more sophisticated services (Kurze et al., 2011). However, hereinafter we focus on the provider perspective. Thus providers can benefit from the increasing capacity and diversity of federated resources. In our model, we employ financial option theory as an interface to elastically allocate an extra pool of federated resources. In finance, an option ${ }^{2}$ is a contract which gives the buyer (the owner) the right, but not the obligation, to buy or sell an underlying asset or instrument at a specified strike price on or before a specified date.

Pricing elasticity and resource trading among federation members lead to competitive contracting process, which aims at finding reasonable and fair price of the asset. The contracting process is to write an option that contains future aspects of trading. For instance, whenever the provider lacks the required resources, then can take advantage of exercising such options to allocate corresponding resources respec-

\footnotetext{
${ }^{2}$ http://en.wikipedia.org/wiki/Option_(finance)
}

tively. Using options, providers take the rights to provision seller's resources which match their demands among parties at a price equal or above to their expectation of the asset payoff. Now, the concern is, how to price an option to be reasonable for both parties? Obviously, option pricing is an elastic process (Dustdar et al., 2011), sensitive to the fluctuation of the asset price determined by supply and demand between federation parties in spot market. As a consequence, pricing elasticity that comes in two types of Demand and Provisioning may drive a wedge between the buying and selling price of an asset. Thus controlling the pricing elasticity of the demand and provisioning with respect to their effects on revenue stream by fair pricing of such options appears to be vital. This paper aims at addressing the pricing elasticity of the asset in federation market by fair pricing of the option. The option price is determined by a broker acting on behalf of the Cloud federation and therefore standardised across the federation. This option gives the right to obtain an instance at a given price, established at the agreement's stipulation time.

In this scenario, at stage 1 as shown in Fig. 1, the clients request for on-demand and RIs and keep using them. At stage 2 another client benefits from the existing RI. As soon as the RI is suspended, Provider $A$ can utilize this instance by reallocating it to unmet on-demand request. Therefore, upon lacking resources, any incoming on-demand request at stage 3 will be responded by reallocation of the RI at 4 to a new client. At this moment, stage 5, Provider A buys an option from federation broker as a supporting mechanism for future resource capacity planning. The provider avoids buying resources at a price that is higher than the one charged to its own customers. As soon as the previous client claims for the RI at 6 which is now allocated to the request 3 , the provider will take advantage of the option signed with Provider $B$ by exercising it at 7 and the Provider $B$ has an obligation to provision the promised resources at 8 . Our focus lies on stages 5 and 7 where the provider is looking for a well priced option to be exercised later to achieve more utilization. In our federation model, Provider $A$ is the demander and Providers $B \& C$ are the resource suppliers in the federated environment.

The fact that future valuation of federated assets depends on the correlated elasticity between provisioning and demand, suggests that the optimal utilization of an asset is primarily driven by its price volatility in open Cloud markets. This influences the trend of providers to be more concentrated on controlling this pricing elasticity. Although the elasticity of a demand is an initial impetus in asset valuation, the pricing elasticity of the demand might lead to ineffi- 


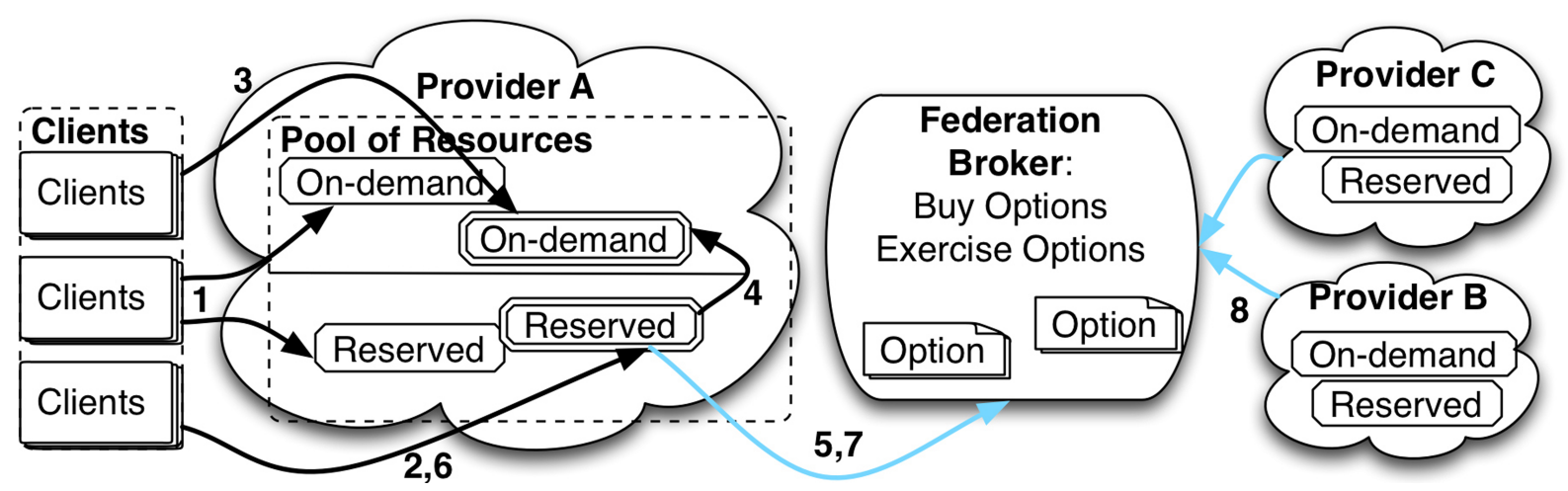

Figure 1: Resource utilization in Cloud federation using options.

cient revenue generation. For instance, the resource demand can be affected to a greater degree by minor changes in asset price. This leads to a question, how can volatility in price cause so much sensitivity in future demands? The reason is amplification. Blame is usually laid on asset price fluctuation and dynamic valuation. The price changes in federation market will be propagated across providers (such as domino effects), causing more sensitivity and concerns on provider's demand. The next question is, how can we control the pricing elasticity and decrease Cloud market sensitivity to future asset price changes? In this study we employ financial option theory which takes care of future valuation of the asset. Then by using the Binomial-Trinomial Tree (BTT) option pricing (shyr Dai and dauh Lyuu, 2010) methodology, we control the Cloud asset price changes and its propagation through the market. As the option price rises or falls, our CAPT model will adjust its structure to the price volatility to come up with an option price that is predictable and fair for both option holder and writer.

\section{TERMS AND PRELIMINARIES}

In this section we present basic concepts, economic terms and numerical methods and their interpretations considered in the study.

\subsection{Cloud Federation Contracts}

In finance, an option is a contract but the major difference arise from the rights and obligations of an option's buyer and seller. A Call option gives the buyer the right, but not the obligation, to purchase the underlying asset at a specified price (the strike or exercise price) during the life of the option. The cost of obtaining this right is known as the option's "premium" which is the price that is offered in the exchange. We use the term premium for an option premium in this study. The option buyer's loss is limited to the premium paid. When you own a Call, what you do by exercising your right is to Call for Resource Provisioning from provider that offered the Call to you. The buyer's right becomes the seller's obligation when the option is exercised. An American option can be exercised at any time during the life of the contract while European option can only be exercised at maturity date. The CAPT is modeled with American call options.

American options are provided by a pool of providers and purchased by other providers as a hedge to cover potential excess demand. Using this method, providers are able to re-sell on-demand VMs that have previously been sold as RI. If the RI owner decides to use the instance then rather than violate an SLA, the excess demand can be covered by exercising previously purchased options to enable Cloud-bursting using the federated pool of resources. The option pricing model determines an option price that is inelastic (such that supply and demand are not highly sensitive to price), thereby reducing self-reinforcing oscillations in supply and demand. The paper demonstrates that the option pricing model converges to a more stable price over time and the simulated provider increases profit from outsourcing provisioning using options.

\subsection{Cloud Asset Pricing Elasticity}

In Cloud systems, elasticity is the ability to automatically increase or decrease resource allocation to asset instances as demand fluctuates. Cloud financial elasticity is a measure of how much resource buyers and sellers respond to changes in market conditions. It's a measure of the responsiveness of quantity demanded or provisioned to a change in one of its determinants like price or quality. In this paper we address the Cloud federation asset pricing elasticity. The law 
of demand states that a fall in the price of a resource raises the quantity demanded. To be more specific, the price elasticity of demand measures how willing providers are to buy less or more options as its price rises or falls. To sum up, the concept of Price Elasticity of Demand (PEoD) measures of how much the resource quantity demanded due to a price change. And the Price Elasticity of Provisioning (PEoP) measures how much the resource quantity provisioned due to a price change (Mankiw, 2012). The PEoD and PEoP formulas are:

$$
\begin{aligned}
& \text { PEoD }=\frac{(\% \text { Change in Quantity Demanded })}{(\% \text { Change in Price })} \\
& P E O P=\frac{(\% \text { Change in Quant ity Provisioned })}{(\% \text { Change in Price })}
\end{aligned}
$$

\subsection{Pricing Elasticity Interpretation}

Regarding interpretation, we analyze the Cloud asset price elasticity only with their absolute values. The PEoD variable values, denote how sensitive the demand for an asset is to a price change. In financial markets, the rule is if a provider's asset has a high elasticity of demand, the more the price goes up, the fewer consumers will buy and try to economise their needs. Correspondingly, in Cloud federation markets, a very high price elasticity suggests that when the price of a resource goes up, our provider will be more sensitive and demand for less assets or buy less call options. Conversely, when the price of that resource goes down, then the provider will demand for more assets or buy more call options. A very low price elasticity implies just the opposite, that changes in price have little influence on demand or exercising the call. To sum up, when demand is price inelastic, total revenue moves in the direction of a price change. When demand is price unit elastic, total revenue does not change in response to a price change. When demand is price elastic, total revenue moves in the direction of a quantity change. In order to see whether the price is elastic or inelastic we use the following rule of thumb:

$$
\text { VM PEoD }=\left\{\begin{array}{cl}
>1 & \text { Demand is price elastic. } \\
=1 & \text { Demand is unit elastic. } \\
<1 & \text { Demand is price inelastic. }
\end{array}\right.
$$

Next is price elasticity of provisioning in federation resource supply pool. The law of supply states that higher prices raise the quantity supplied. The price elasticity of supply measures how much the quantity supplied responds to changes in the price. Supply of a good is said to be elastic if the quantity supplied responds substantially to changes in the price. Supply is said to be inelastic if the quantity supplied responds only slightly to changes in the price. PEoP denotes how sensitive the provisioning of an asset is to a price change. In Cloud federation markets, a very high price elasticity of provisioning suggests that when the price of a resource goes up, Cloud federation members will be more sensitive to price changes and provision more assets or sell more call options to make more profit. Thus, the resource quantity supplied can respond substantially to price changes. Same as PEoD, in order to see whether the price is elastic or inelastic in PEoP, we use the following rule of thumb:

$$
\text { VM PEoP }= \begin{cases}>1 & \text { Provisioning is price elastic. } \\ =1 & \text { Provisioning is unit elastic. } \\ <1 & \text { Provisioning is price inelastic. }\end{cases}
$$

Finding the right balance between these two polar approaches of PEoD and PEoP to come to a new equilibrium is a challenge as we address it using our CAPT model. In equilibrium, asset aggregate demand has to equal the asset supply. To be more specific, in our evaluation, we will show that our pricing model, calculates the fair price of the option that makes the demand, price inelastic and provisioning, price elastic. This leads to increasing demand, regardless of the asset price oscillation.

\subsection{Assumptions}

It is an indication that the following assumptions underlying our model has been considered for the proper positioning of this study. In a Cloud market, resources are virtualized to abstract concepts like virtual machines (VMs) and assumed as intangible assets. They are also seen as assets as long as associated with a contract that can be exercised by an option. Federation formation pose some concerns like contract management, data policies, SLA violations and etc. We believe these concerns should be addressed in the business models agreed among parties.

\section{CAPT MODEL}

The option pricing can be represented by numerical methods like trees. This section shows how to generate the CAPT tree for pricing options. The model benefits from the Binomial and Trinomial tree methods as detailed below. This section shows how to generate the CAPT tree for pricing options. 


\subsection{Binomial Tree}

Binomial tree model is a numerical pricing method that approximates option price. Let a derivative on $S_{(t)}$ initiates at time 0 and matures at time $T$. A lattice partitions this time span into $\mathrm{n}$ equal-distanced time steps and specifies the value of $S_{(t)}$ at each time step which denotes the Cloud asset price. Let the length between two adjacent time steps be $\Delta t \equiv T / n$. The established Cox-Ross-Rubinstein (CRR) binomial tree (Cox et al., 1979) is shown in Fig. 2. As we move forward in time, each asset price $S$ can either move upward to become $S_{u}$ with probability $P_{u}$, or move downward to become $S_{d}$ with probability $P_{d} \equiv 1-P_{u}$. The CRR lattice adopts the following solution:

$u=e^{\sigma \sqrt{\Delta t}}, d=e^{-\sigma \sqrt{\Delta t}}, P_{u}=\frac{e^{r \Delta t}-d}{u-d}, P_{d}=\frac{e^{r \Delta t}-u}{d-u}$

Where $\sigma$ is price volatility, $\Delta t$ is duration of a step and $r$ denotes the interest rate.

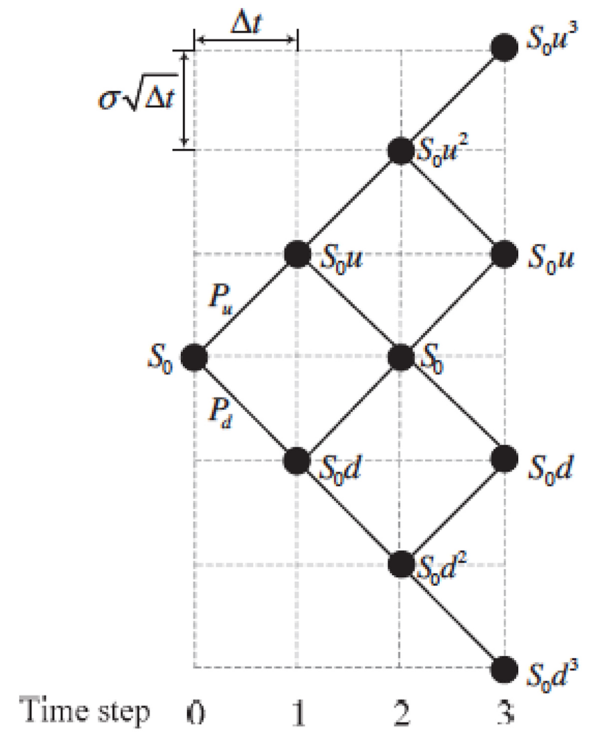

Figure 2: CRR Binomial Pricing Tree.

\subsection{Trinomial Tree}

A trinomial tree can be built in a similar way to the binomial tree but has three possible paths (up, down, and stable) per node leading to more efficient pricing. The jump sizes $(u, d)$ can be calculated in a similar way with doubled time spacing. The transition probabilities are given as:

$P_{u}=\left(\frac{e^{\frac{r \Delta t}{2}}-e^{-\sigma \sqrt{\frac{\Delta t}{2}}}}{e^{\sigma \sqrt{\frac{\Delta t}{2}}}-e^{-\sigma \sqrt{\frac{\Delta t}{2}}}}\right)^{2} P_{d}=\left(\frac{e^{\sigma \sqrt{\frac{\Delta t}{2}}}-e^{\frac{r \Delta t}{2}}}{e^{\sigma \sqrt{\frac{\Delta t}{2}}}-e^{-\sigma \sqrt{\frac{\Delta t}{2}}}}\right)^{2}$

$$
P_{m}=1-P_{u}-P_{d}
$$

Now it is possible to find the value of the underlying asset, $S$ for any sequence of price movements. It will generate a directed graph with nodes labeled as asset prices and edges connecting nodes separated by one time step and a single price up, down and middle jumps as $N_{u}, N_{d}, N_{m}$, where the price after $i$ period at node $j$ (or after $i$ ups and $j$ downs) is given by: $S_{(i, j)}=u^{N_{u}} d^{N_{d}} m^{N_{m}} S\left(t_{0}\right)$ where $N_{u}+N_{d}+N_{m}=i$. Finally, in both binomial and trinomial tree methods, the option value can be computed by standard backward induction method.

\subsection{Growing the CAPT Tree}

This section visualizes how the BTT tree is constructed for pricing the options briefly. In this model as illustrated in Fig. 3 the root of the tree is the node $\mathrm{S}$ which is formed by a trinomial tree and the rest of the tree is constructed using binomial method with the first two time steps truncated. The barriers (the black nodes) are $H_{0}$ and $L_{0}$ at time $T_{0}$ and $H_{1}$ and $L_{1}$ at time $T_{0}+T_{1}$. These barriers define the allowable range for the price fluctuation of the underlying asset serving to limit both, profits and losses, for federation parties. The tree adjusts and adapts its structure to the price volatility and the moving barriers to come up with an option price that is predictable and fair for both option holder and writer.
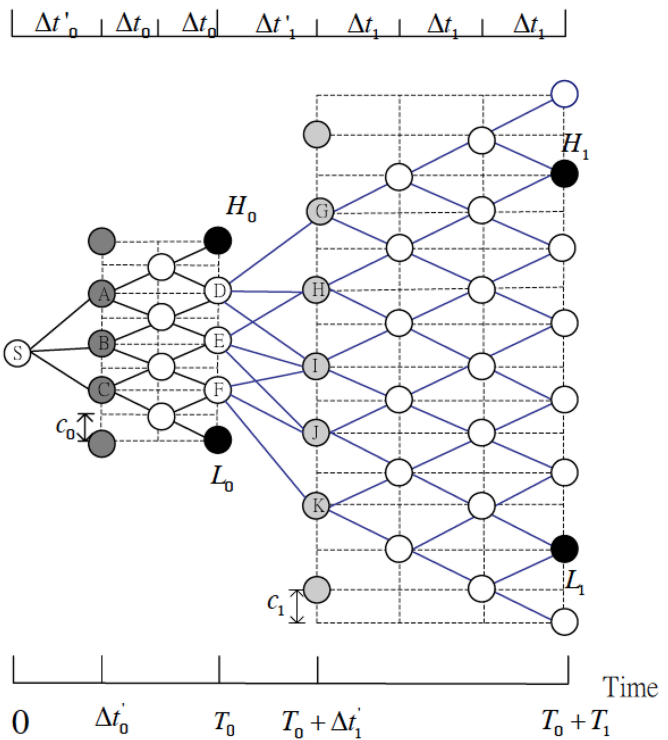

Figure 3: Cloud Option Pricing using Bino-Trinomial Tree.

The combinatorial pricing algorithm (Dai and Lyuu, 2007) is used to evaluate the option values on the three CRR trees as shown in Fig. 3, with root nodes A, B, and C. The option price of the CAPT at 
node $S$ is also evaluated by the backward induction method.

\section{Model Evaluation}

Now, we present results from our simulation observation that show the efficiency of our model. We have implemented a Cloud federation environment using Cloud simulation platform, CloudSim (Calheiros et al., 2011). The simulated Cloud federation uses our option pricing model for trading assets and VM provisioning. The unit of resource being observed is an Amazon EC2 Standard Small Instance (US East). At the date of simulation (Sept 2013), resources advertised at a price of $\$ 0.085 /$ hour for an on-demand instance. For RIs, the same type instance for 12 months costs $\$ 0.034 /$ hour. For evaluation purposes, (i) the reserved capacity of the data center is considered as steady constant value during simulation. (ii) to economize the equations, we do not take into account the operational costs (i.e., hardware and software acquisition, staff salary, power consumption, cooling costs, physical space, etc.) of the data center. It imposes a constant value within the model.

\subsection{Simulation Setup}

The simulation environment is developed to capture the behavior of our CAPT model in Cloud federation where supply and demand fluctuate in daily patterns directly inspired by real-world market. For a provider, who benefits from this market, simulation was implemented with a resource pool capable of 400 simultaneous running VMs capacity including reserved and on-demand. We have implemented the following three components on top of CloudSim simulator. Further details of our option-based federation simulator entities and settings are as follows.

\subsubsection{CAPT Request Generator (ReqG)}

The workload pattern generation was needed in order to mimic the real world IaaS Cloud requests. We implemented CAPT-ReqG agent to create jobs by using the Cloudlet class in CloudSim. In our model, each job has an arrival time as we scheduled the workload on a daily-basis pattern and a duration time which is the holding time of the instance by the job and metered to charge the consumer respectively. Given that our workload follows daily pattern based on normal Gaussian distribution for the 24 hours of a day and considering standard business hours (from 9 to 17) as peak hours, we generate a randomly distributed arrival time for requests in each specific hour. The load decreased $60 \%$ on weekends.

\subsubsection{CAPT Resource Allocator (ResA)}

We have developed the CAPT-ResA agent to determine the association between jobs and federated resources. Our allocation policy finds a mapping between the batch of jobs outsourced to the federation and VMs associated to options. In the simulation, the VM provisioning policy is extended to best fit with respect to the option status. The providers are implemented using DataCenter class in CloudSim, as it behaves like an IaaS provider. The CAPT-ResA receives requests from $C A P T-\operatorname{Req} G$, allocates resources and binds the jobs to the VMs accordingly. For resource allocation, we have used shared pool strategy. In case of arriving a new on-demand job, the agent checks if the number of currently running on-demand jobs exceeds the capacity of on-demand pool and if so, it will allocate VMs from its reserved pool while buying an option from federated Cloud. As soon as it receives requests which can not be met in-house, the agent will exercise the options that were bought before and outsource the new jobs to federated pool.

\subsubsection{CAPT Option Handler (OptH)}

Our $C A P T-O p t H$ agent implements the option pricing model as detailed in section 4 . It also routes the option exercising request to the CAPT-ResA agent to have the requested resource provisioned. The pricing policy is set to resource/hour consumed for each instance, for the duration an instance is launched till it is terminated. Each partial resource/hour consumed will be charged as a full hour. There are six metrics that affect the CAPT option pricing, (i) the current stock price, $S_{0}$ set to $\$ 0.034 /$ hour (ii) the exercise price (spot price), $K$ is generated based on Amazon spot price observed pattern. (iii) the time to option expiration $T$ set to 1 month (iv) the volatility $\sigma$ which is the range and speed in which a price moves, set to $31.40 \%$ per annum. It is observed by the cloudexchange.org ${ }^{3}$ which is real-time monitoring of Amazon EC2 spot prices. (v) the interest rate, $r$ is set to $19.56 \%$ per annum since the Amazon EC2 SLA ${ }^{4}$ interest rate is $1.5 \%$ per month and (vi) the dividend expected during the life of the option is set to $\$ 5.17$. Both High and Low barriers are set to \$0.039 and $\$ 0.030$. The simulation was run 50 times. The experiment duration is set to 6 months and the mean value

\footnotetext{
${ }^{3} \mathrm{https}: / /$ github.com/tlossen/cloudexchange.org

${ }^{4}$ http://aws.amazon.com/agreement/
} 
of the results is evaluated to mimic the real-world environment.

\subsection{Evaluation Measure}

There are four empirical measures as we care to specify and observe their behavior during the simulation: (i) Provider's profit $\left(P_{p r}\right)$, which our model claims to ensure the optimal utilization of the resources for providers. The profit measurement equation is:

$$
P_{p r}=R_{o n}+R_{\text {res }}+R_{(\text {op }, \text { exe })}-C_{(\text {op }, \text { pre })}-C_{(\text {gen })}
$$

where $R_{o n}$ and $R_{\text {res }}$ are the providers' total revenue received over their own on-demand and RIs. $R_{(o p, e x e)}$ is the revenue of exercising the options since the option exercise price is less than their own instance price sold to their clients before. $C_{(o p, p r e)}$ denotes the premium to be paid for the purchase of the option which our model calculates accurately. Finally, $C_{(g e n)}$ covers general costs of provider as we assumed a constant value. (ii) Second measure is $Q o S$ Violations $\left(Q o S_{v}\right)$, that holds the number of rejected or unmet reserved and on-demand instances reflecting the SLA violations. Third and forth measures are (iii) Price Elasticity of Demand $\left(\varepsilon_{D}\right)$ and (iv) Price Elasticity of Provisioning $\left(\varepsilon_{P}\right)$ where their absolute values are highly correlated with the asset price changes. Their computation is done with these equations:

$$
\varepsilon_{D}(v m)=\frac{\% \Delta_{Q d}}{\% \Delta_{P_{v m}}} \text { and } \varepsilon_{P}(v m)=\frac{\% \Delta_{Q p}}{\% \Delta_{P_{v m}}}
$$

The $\varepsilon_{D}(v m)$ and $\varepsilon_{P}(v m)$ denote the price elasticity of demand and provisioning of an asset, and measures the percentage change in the quantity of VM demanded and provisioned per $1 \%$ change in the price of its option premium. Our economic model should make $\varepsilon_{D}(v m)$ "price inelastic" and $\varepsilon_{P}(v m)$ "price elastic" as interpreted in section 3.2.

\subsection{Results and Debate}

The aggregate results imply utility and are reported as summary in Table 1. Results show that those providers are able to reach an utilization rate of $99 \%$ and achieve gains both from the in-house instances and from those obtained by exercising the option's rights from other providers of the federation. Taking these results together, four points stand out in this simulation. First, is the profit made from exercising options. To interpret this, note that in our approach, providers buy the options that its exercise price are less than their own VM provisioning price. As observed, providers were able to meet $86 \%$ of an incoming requests by inhouse provisioning and outsourced
$14 \%$ of their demands to the federation, in which are fully provisioned to celebrating $8.7 \%$ more profit. Second, is the achievement over the QoS agreed with the client for resource delivery. For both reserved and on-demand, no QoS violation (no unmet request) is detected. Third, as our results indicate, the value of pricing elasticity of demand (PEOD) is kept less than 1 denoting that the demand became "price inelastic" serving to increasing demand, regardless of the asset price oscillation. From the federation perspective (resource suppliers side), the value of pricing elasticity of provisioning $(P E O P)$ is more than 1 denoting that the provisioning became "price elastic" indicating the providers are flexible enough to adapt the amount of resources they provision. These values are consistent with the number of options purchased and exercised, leading to more economies of scale. Finally, is the utilization value, which is considerable. This indicates that optimal utilization of resources is achieved to exploit the efficiency and accuracy of our model.

To form a basis for comparison, our next two figures depict the dependencies between option pricing elasticity and its demand and provisioning. Fig. 4, shows how CAPT controls the option pricing elasticity and converges to a more stable price smoothly. Our approach finds the optimal option price of the federated resource in the Cloud to come to an equilibrium between PEoD and PEoP. The asset equilibrium price occurs when the supply resource pool matches the aggregate demand indicating an optimal resource utilization. From the provisioning perspective, as shown in Fig. 5, it can be seen, that a $21 \%$ increase (using midpoint method) in the asset price leads to a $35 \%$ increase in quantity provisioned. This indicates an elastic supply. Since the asset pricing elasticity is controlled, we see a synchronous correlation between price and supply changes. As a result, the total revenue moves in the direction of price change.

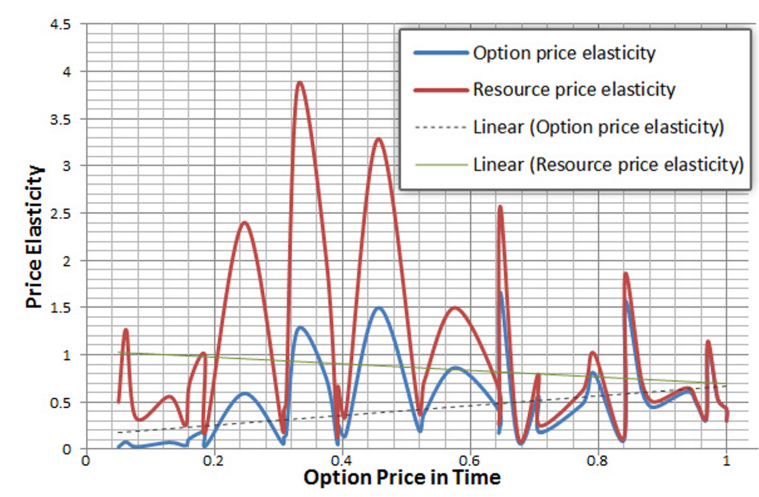

Figure 4: Resource price elasticity controlled via options. 
Table 1: Cloud federation market simulation summary (6 months).

\begin{tabular}{|c|c|c|c|c|c|c|c|c|c|c|}
\hline \multirow[b]{2}{*}{ Market $\downarrow$ Measures $\rightarrow$} & \multirow[b]{2}{*}{ Workload } & \multirow[b]{2}{*}{ Utilization } & \multicolumn{2}{|c|}{ QoS Violations } & \multicolumn{2}{|c|}{ Profit } & \multicolumn{2}{|c|}{ Price Elasticity } & \multicolumn{2}{|c|}{ Options } \\
\hline & & & Reserved & $\overline{\text { On-demand }}$ & In-house & Option & PEoD & PEoP & Bought & Exercised \\
\hline Cloud Federation Market & 98455 & $99 \%$ & 0 & 0 & 35293.63 & 3339.97 & 0.095 & 1.28 & 25676 & 14020 \\
\hline
\end{tabular}

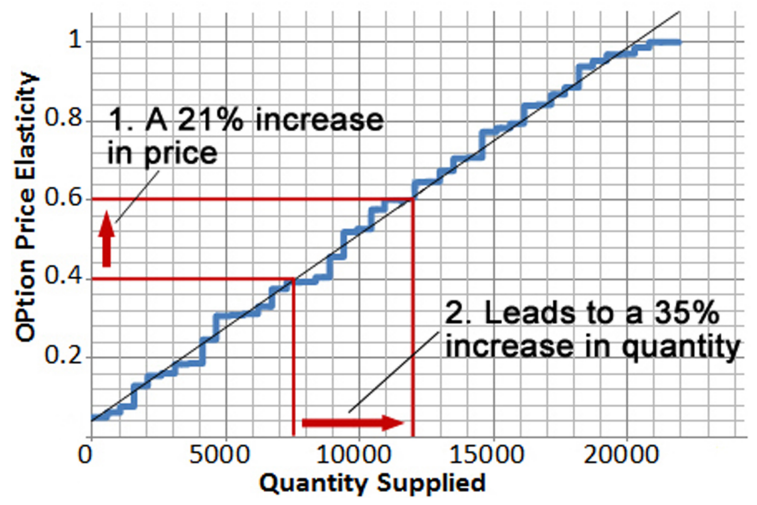

Figure 5: Price elasticity of resources provisioned.

\section{RELATED WORK}

In relation to our approach, there are some alternatives that propose federation economic model more focused on the provider's perspective. A broker-based federation approach has been proposed by (Villegas et al., 2012), (Rogers and Cliff, 2012) and (Raj, 2011). These studies decouple the brokerage strategies and federation economic valuation. Zhang proposes an economic model for the evaluation of the economic value of Cloud Computing Federation in providing one computing unit such as the power and human resources (Zhang and Zhang, 2012). Just as Clouds enable users to cope with unexpected demand loads, a Federated Cloud will enable individual Clouds to cope with unforeseen variations of demand. Authors in (Gomes et al., 2012) investigate the application of market-oriented mechanisms based on the General Equilibrium Theory of Microeconomics to coordinate the sharing of resources between the Clouds in the federated environment. In (Zhao et al., 2012), authors present an online resource marketplace for open Clouds by adopting an eBay style transaction model based on auction theory. Here (Samaan, 2013) establishes a novel economic sharing model to regulate capacity sharing in a federation of hybrid Cloud providers. The idea of financial options is used by (Sharma et al., 2012) as a financial model for pricing Cloud compute commodities by using Moore's law on depreciation of asset values, to show the effect of depreciation of Cloud resource on QoS. In (Toosi et al., 2012) authors incorporate financial options as a market model for federated Cloud environments. In con- trast to existing approaches, we use financial option theory for asset trading and propose a dynamic and adaptive option pricing model which enhance profit by controlling the pricing elasticity of demand and provisioning in the Cloud federation.

\section{CONCLUSION AND OUTLOOK}

Providers consider federations as an alternative pool of resources to their expected consumption growth. Their demand to use the federated asset is dependent to the pricing elasticity of demand, as if the elasticity is high, then they will be more careful on buying options. In this paper, we proposed a financial option pricing model to address the pricing elasticity concerns in above situation. Our economical model is for implementing a future market of virtualized resources in a system where a federation of Cloud providers is used to reduce risks and costs associated with the capacity planning of Cloud providers. Providers will benefit by this model to make decisions when to buy options in advance and when to exercise them to achieve more economies of scale.

So far, we have proposed an economic model that considers future aspects of trading like capacity planning or resource allocation over upcoming demands. The CAPT model empowers vendors to get additional resources as and when required. This economic model aims for the leverage of demand and supply form the IaaS provider and third party providers point of view, finding suboptimal price policies between resources ownered by the provider and options to external providers using Cloud bursting when needed. This study covers two aspects of resource elasticity: Resource Quantity and Price. As an outlook, our future work includes further extension to the model that can also support the Quality of Service $(Q o S)$ aspect in federation environment. 


\section{REFERENCES}

Calheiros, R. N., Ranjan, R., Beloglazov, A., De Rose, A. F., and Buyya, R. (2011). Cloudsim: a toolkit for modeling and simulation of cloud computing environments and evaluation of resource provisioning algorithms. Softw. Pract. Exper., 41(1):23-50.

Cox, J. C., Ross, S. A., and Rubinstein, M. (1979). Option pricing: A simplified approach. Journal of Financial Economics, 7(3):229-263.

Dai, T.-S. and Lyuu, Y.-D. (2007). An efficient, and fast convergent algorithm for barrier options. In Kao, M.Y. and Li, X.-Y., editors, Algorithmic Aspects in Information and Management, volume 4508 of Lecture Notes in Computer Science, pages 251-261. Springer Berlin Heidelberg.

Dustdar, S., Guo, Y., Satzger, B., and Truong, H.-L. (2011). Principles of elastic processes. Internet Computing, IEEE, 15(5):66-71.

Gomes, E. R., Vo, Q. B., and Kowalczyk, R. (2012). Pure exchange markets for resource sharing in federated clouds. Concurr. Comput. : Pract. Exper., 24(9):977991.

Kurze, T., Klems, M., Bermbach, D., Lenk, A., Tai, S., and Kunze, M. (2011). Cloud federation. Computing, (c):32-38.

Mankiw, N. G. (2012). Elasticity and its applications. In Principles of Microeconomics, 6th Edition, pages 89109. Harvard University.

Raj, G. (2011). An efficient broker cloud management system. In Proceedings of the International Conference on Advances in Computing and Artificial Intelligence, ACAI '11, pages 72-76, New York, NY, USA. ACM.

Rogers, O. and Cliff, D. (2012). A financial brokerage model for cloud computing. Journal of Cloud Computing, 1(1):1-12.

Samaan, N. (2013). A novel economic sharing model in a federation of selfish cloud providers.

Sharma, B., Thulasiram, R., Thulasiraman, P., Garg, S., and Buyya, R. (2012). Pricing cloud compute commodities: A novel financial economic model. In Cluster, Cloud and Grid Computing (CCGrid), 2012 12th IEEE/ACM International Symposium on, pages 451457.

shyr Dai, T. and dauh Lyuu, Y. (2010). The bino-trinomial tree: A simple model for efficient and accurate option pricing. Journal of Derivatives, 17(4):7-24.

Toosi, A., Thulasiram, R., and Buyya, R. (2012). Financial option market model for federated cloud environments. In Utility and Cloud Computing (UCC), 2012 IEEE Fifth International Conference on, pages 3-12.

Villegas, D., Bobroff, N., Rodero, I., Delgado, J., Liu, Y., Devarakonda, A., Fong, L., Masoud Sadjadi, S., and Parashar, M. (2012). Cloud federation in a layered service model. J. Comput. Syst. Sci., 78(5):1330-1344.

Zhang, Z. and Zhang, X. (2012). An economic model for the evaluation of the economic value of cloud computing federation. In Zhang, Y., editor, Future Communication, Computing, Control and Management, volume
141 of Lecture Notes in Electrical Engineering, pages 571-577. Springer Berlin Heidelberg.

Zhao, H., Yu, Z., Tiwari, S., Mao, X., Lee, K., Wolinsky, D., Li, X., and Figueiredo, R. (2012). Cloudbay: Enabling an online resource market place for open clouds. In Utility and Cloud Computing (UCC), 2012 IEEE Fifth International Conference on, pages $135-142$. 